QVIPURAMAYOC $\mid$ Revista de la Facultad de Ciencias Contables

Vol. 20 N.o 38 pp.65-74 (2012) UNMSM, Lima, Perú

ISSN: 1560-9103 (versión impresa) / ISSN: 1609-8196 (versión electrónica)

\title{
EFICACIA DEL PRESUPUESTO POR RESULTADOS EN EL MARCO DE LA PLANIFICACIÓN ESTRATÉGICA \\ MULTIANUAL
}

\section{EFFECTIVENESS OF THE BUDGET BY RESULTS UNDER} THE MULTI-YEAR STRATEGIC PLANNING

\author{
LeOnCIO Javier Melgarejo * \\ Docente Asociado de la Facultad de Ciencias Contables \\ Universidad Nacional Mayor de San Marcos-UNMSM \\ Lima-Perú
}

[Recepción: Setiembre de 2012/ Conformidad: Octubre 2012]

\section{RESUMEN}

Con la globalización del mundo EconómicoFinanciero y los consiguientes tratados de Libre Comercio en especial con Estados Unidos, sustentada en la solvencia de la Liquidez acorde al Manual de Estadística de Finanzas Publica Mundial se ha impuesto en nuestro País la Corriente Financista. En tanto, desde los inicios del milenio se viene implementándose una Nueva Estructura de la Administración Financiera del Estado Peruano, que se yergue sobre cuatro sistemas nacionales estratégicos.

En este contexto: Los nuevos Clasificadores Presupuestarios, Plan de Cuentas, Programación Multianual de la Inversión Pública "PMIP", Programación del Calendario Anual "PCA" y Presupuesto por Resultados (PpR), coadyuvante con el cuarto objetivo del Plan Estratégico 2009-2011 del CEPLAN: "Articulación del Plan con el Presupuesto por Resultados"; son dadas en miras de lograr mayor Beneficio Social. Aunque, el Plan Estratégico Nacional denominado como PLAN BICENTENARIO-PERU HACIA 2021, todavía sigue en suspenso y aún no se implementa el Plan Operativo Nacional, hace que nuestro País sigue técnicamente navegando sin brújula. La aplicación del Presupuesto por Resultados, pese su poca dinámica, ya puede resultar efectiva con las proyecciones plurianuales de la Inversión Pública y Programaciones de Compromisos Anuales financiándose inclusive con los recursos patrimoniales. La oportunidad está en la Gestión Gerencial

Palabras Clave: Planificación, presupuesto, gestión, beneficio.

\section{ABSTRACT}

With the globalization of the economic and financial world, and particulary with the subsequent free trade agreements with the United States, supported in the solvency of the liquidity, according to statistical manual of global public finance, has been imposed in our country, the financial currents. Meanwhile, since the beginning of the millennium has been implemented a new financial framework of the financial management structure of the Peruvian state that is standing on four strategic national systems.

In this context: The new classifiers of budget, Plan of Accounts, Programming Multi-Year Public Investment "PMIP", Programming the Annual Calendar "PCA" and performance budgeting (PPR), in contribution with fourth objective of the Strategic Plan 2009-2011 CEPLAN: "Articulation of the Plan with performance budgeting", are given in order to achieve greater social benefit. Although, the National Strategic Plan called as PLAN BICENTENNIAL-PERU GO TO 2021, is still pending and not yet has been implemented the National Operational Plan, our country is still technically sailing without a compass.

The implementation of performance budgeting, despite its low dynamics, and can be effective with multiyear projections of Public Investment and programmings of annual commitments financed even with heritage resources. The opportunity lies in business management.

Keywords: Planning, budgeting, management, benefit

* Contador Público Colegiado. E-mail: jmelgarejo@hotmail.com 


\section{INTRODUCCIÓN}

En la Gestión Económica-Financiera el rol del Presupuesto es preponderante y como tal ya se encuentra firmemente sistematizado desde 2003, con la autonomía propia del Órgano Rector: Dirección Nacional del Presupuesto Público y como pilar fundamental del Sistema de la Administración Financiera del Sector Público. Que en busca de lograr los objetivos del Estado, con una Administración Pública eficiente, oportuno, eficaz y capaz es que desde 2008 con la dación de la Ley 28927- Ley del Presupuesto del Sector Público se viene implementando el Presupuesto por Resultados.

No hay Presupuesto, sin la debida programación de actividades y metas y por ello, inclusive se conceptúa al Presupuesto Analítico Institucional (PAI) como el financiamiento del POI. En tanto Siendo este instrumento como todo PLAN parte del proceso del Quehacer científico de Ciencias Administrativas en el presente estudio se analizará la problemática de la vigencia de Planificación con la consiguiente deficiente aplicación del Presupuesto por Resultados. Es el caso en el campo de Planificación aun el Sistema no funciona, pese que desde 1962 mediante Decreto Ley 14220 se creará el Sistema Nacional de Planificación del Desarrollo y Social, estableciéndose en 1981 como órgano Rector al Instituto Nacional de Planificación (INAP), pero que en 1992 se desactivara pasando sus funciones al MEF, hasta que en 2002 en las mesas del tratado de Acuerdo Nacional se diera su gran importancia del Planeamiento Estratégico para alcanzar los objetivos nacionales de Desarrollo y Crecimiento Integral en la Economía Globalizada, lográndose en el año 2003 se constituyera una Comisión Organizadora y en 2005, por Ley 28522 se dio la Ley del Sistema Nacional del Planeamiento Estratégico y Centro de Planeamien- to Estratégico "CEPLAN, pero que no tuvo su debida vigencia hasta que nuevamente en el año 2008 mediante del Decreto Legislativo 1088 (28-06-08) se diera la nueva Ley con el mismo nombre e institución rectora pero adscrita al PCM. De cuya labor resulto el PLAN BICENTENARIO del Perú, cuya vigencia se encuentra suspendida. Que indudablemente retrasa la contundente aplicación del Presupuesto por Resultados.

En tanto sigue las preguntas porqué el Estado, no cumple con su Misión, porqué las normas no contribuyen totalmente con la eficacia y calidad de gasto público en la medida que no está orientado a la planificación consecución y medición de resultados. En nuestro país, el manejo presupuestario público se ha caracterizado por ser inadecuado y presentar evidentes problemas en cuanto a la eficacia y calidad del gasto. En consecuencia, la provisión de bienes y servicios a la población ha sido, generalmente, deficiente y poco oportuna. Pese a ello, año tras año, los presupuestos institucionales se mantienen en la misma estructura de asignación de recursos. Para una gestión rutinaria sin cambios ni resultados, con un proceso de formulación que difiera significativamente de la realidad convirtiéndose en asignaciones verticales del MEF con:

- Desarticulación entre planificación y presupuesto.

- Asignación de recursos centrada en insumos antes que en productos y resultados.

- Poca flexibilidad para adecuar las estrategias a los cambios que se van produciendo.

- Excesiva atención al control del proceso presupuestario antes que en los resultados.

- Lógica del ajuste y de la austeridad mal enfocada.

Por esta situación, con el presente estudio luego de haber tratado las causas de su postergación y pocos logros de los benefi- 
cios en la aplicación del $\mathrm{PpR}$, se recomienda que para la efectividad de la Administración Económica Financiera del Sector Público debe tomarse en cuenta la vigencia de la Programación de Compromisos Anuales en relación al Plurianual Presupuestario y la Multianualidad de las Inversiones Públicas, que están vigentes mientras se consolide el Sistema Nacional de Planificación

\section{MARCO TEORICO: NUEVA ESTRUCTURA DE LA AMDINISTRACIÓN FINANCIERA DEL ESTADO}

\section{CONCEPTOS BÁSICOS}

PRESUPUESTO: Financiamiento y aplicación de los Recursos Públicos al Plan Operativo Institucional.

PLAN OPERATIVO INSTITUCIONAL: conjunto de actividades, con indicadores de metas del ejercicio fiscal, relacionados a la misión institucional.

FONDOS PÚBLICOS.- Todos los recursos financieros de carácter tributario y no tributario que se generan, obtienen u originan en la producción o prestación de bienes y servicios que las unidades ejecutoras o entidades realizan, con arreglo a Ley.

Los provenientes de la recaudación tributaria nacional así como de aquellos ingresos no tributarios, son administrados y registrados por la Dirección Nacional del Tesoro Público; mientras los generados u obtenidos en la producción o prestación de los bienes y servicios autorizados con arreglo a Ley, son administrados y registrados por las unidades ejecutoras y entidades que los generan.

CAJA ÚNICA.- A cargo de la Dirección Nacional del Tesoro Público, constituida por la Cuenta Principal y las otras cuentas bancarias de las cuales es titular. Asimismo forman parte de la Caja Única las cuentas bancarias donde se manejan fondos públicos, cualquiera que sea su fuente de financiamiento, finalidad o entidad titular de las mismas.

No forman parte de la Caja Única las cuentas bancarias de ESSALUD y de aquellas entidades constituidas como personas jurídicas de derecho público y privado facultadas a desarrollar la actividad empresarial del Estado.

CUENTA PRINCIPAL.- Llamada como CUENTA UNICA, es una cuenta bancaria ordinaria aperturada en el Banco de la Nación, en la que se centraliza a nombre de la Dirección General del Tesoro Público; quien autoriza la apertura de:

Subcuentas Bancarias para la recaudación de Ingresos.

Subcuentas Bancarias de Gastos para registrar operaciones de pagaduría que se realizan sobre la base de autorizaciones aprobadas por la DNTP en proceso de ejecución de gasto de acuerdo a Ley. Se apertura a nombre de las correspondientes Unidades Ejecutoras o Entidades, las mismas que son directamente responsables de su manejo.

\section{SISTEMATIZACIÓN DE LA ADMINISTRACIÓN FINANCIERA DEL ESTADO PERUANO}

Luego de diferentes ensayos de mejora de la Gestión Económica Financiera del Estado Peruano, mediante Ley 28112 (27-11-2003) se dio LEYMARCO DE LAADMINISTRACION FINANCIERA DEL SECTOR PUBLICO, sustentado en cuatro (4) sistemas fundamentales según la corriente financistas para la Administración Económica-Financiera del Estado Peruano, que consiguientemente se dieron con sus propias leyes orgánicas y sus correspondientes Principios, como son: 
1. Mediante Ley 28411(25-11-2004): Sistema Nacional de Presupuesto Público, con su órgano Rector: Dirección General del Presupuesto Público DGPP. y sus catorce (14) PRINCIPIOS REGULATORIOS:

- Equilibrio presupuestario,

- Equilibrio macro fiscal

- Especialidad cuantitativa

- Especialidad cualitativa

- Universalidad y unidad

- De no afectación predeterminada

- Integridad

- Información y especificidad

- Anualidad

- Eficiencia en la ejecución de los fondos públicos

- Centralización normativa y descentralización operativa

- Transparencia presupuestal

- Exclusividad presupuestal

- Principio de Programación Multianual

Además con dos principios complementarios: Legalidad y el de presunción de veracidad, de conformidad con la Ley Marco de la Administración Financiera del Sector Público.

2. Mediante Ley 28693 (21-03-2006) Sistema Nacional de Tesorería, con su Órgano Rector Técnico-Normativo: Dirección General del Tesoro Público y con sus siete

(7) Principios:

- Unidad de Caja,

- Economicidad

- Veracidad,

- Oportunidad,

- Programación

- Seguridad
3. Mediante Ley 28708 (02-04-2006): Sistema Nacional de Contabilidad, Ley que sustituyera a la Ley 24680 ( 04-05- 1987) con lo que se había implementado la Contaduría de la Nación, sustituyéndose por la Dirección General de Contabilidad Pública como Órgano Rector Técnico- Normativo en asuntos de Contabilidad DGNCP y con sus cinco (5): PRINCIPIOS REGULATORIOS

- Uniformidad

- Integridad

- Oportunidad

- Transparencia

- Legalidad

4. Mediante Ley 28563 (30-06-2006): Sistema Nacional de Endeudamiento Público, con su Órgano Rector: Dirección Nacional de Crédito Público y sus cuatro (4) PRINCIPIOS REGULATORIOS:

Eficiencia y Prudencia.- Basada en una estrategia de largo plazo, para cubrir parte de los requerimientos de financiamiento del sector público a los más bajos costos posibles, sujetos a un grado de riesgo prudente y en concordancia con la capacidad de pago del país.

Responsabilidad Fiscal.- El endeudamiento público debe contribuir con la estabilidad macroeconómica y la sostenibilidad de la política fiscal, con reglas y límites prudentes. En tanto, el Gobierno Nacional no reconocerá deudas contraídas por los Gobiernos Regionales y Locales, salvo las debidamente avaladas.

Transparencia y Credibilidad.- El proceso de endeudamiento debe llevarse a cabo mediante mecanismos transparentes y pre- 
decibles previstos en la Ley (Del Endeudamiento Anual)

Capacidad de Pago.- De la entidad beneficiada.

\section{PRESUPUESTO POR RESULTADOS}

Es una modalidad por la cual se identifica productos que ofrece un Pliego Presupuestario combinando y costeando los insumos (bienes y servicios) que se traducen en productos, orientados a lograr resultados en la Ciudadanía.

Con esta implementación se trata de implementar la Gestión Gerencial , otorgándose una mayor flexibilidad para el cumplimiento no solamente metas sino también objetivos en busca de los fines que busca el Estado: Bienestar de la Sociedad al que representa de manera organizada para satisfacer sus necesidades para lo cual contribuye mediantes sus tributos.

En tanto las programaciones de actividades mediante el Plan Operativo Institucional POI en relación del Plan Estratégico Institucional PEI que inclusive va más allá de la duración de un gobierno. De acuerdo a esta Programación o Planificación Multianual de actividades, proyectos, programas, etc. También el Presupuesto se formula en una programación a largo plazo en el marco de las políticas Macroeconómicas del Estado, que permita el comportamiento fiscal más allá de la coyuntura del momento, equivalente con los proyectos que se formulan y se direccionan bajo la normatividades y competencia de la Dirección General Multianual,

Este proceso se implementa en nuestro país desde el año 2007 que por Ley 28927 Presupuesto del Sector Público se dio la responsabilidad a la DGPP, diseñar e implementar la Gestión Presupuestaria por Resultados a fin dar respuesta a:
La baja ejecución de los recursos asignados.

Descuidos politizados o burocráticos para gastar antes del fin de cada período en compras no alineadas a los servicios que requieren prestar las entidades.

El objetivo fundamental es cumplir con la misión del Estado buscar beneficios de la ciudadanía con una administración pública eficaz e eficiente con criterios de gestión de los productos con el uso apropiado de los bienes y servicios. En tanto través de la Ley 28927- Ley del Presupuesto del Sector Público para el Año Fiscal 2008-, Capítulo IV, se incorporó los elementos básicos para la introducción del Presupuesto por Resultados en el Perú, estableciendo una ruta para su progresiva implementación en todas las entidades de la administración pública peruana y en todos los niveles de gobierno. Así como a partir del 2009 la implementación del Presupuesto Multianual de Inversión Pública. Sin embargo en el año 2010 aún se encomendó a la DGPP, seguir diseñando su implementación en ciertos programas socioeconómicos y así en las siguientes leyes de presupuestos ciertas deposiciones mediatecas.

El Presupuesto por Resultados es llegar a la Sociedad con enfoque institucional, con una visión integrada de planificación y presupuesto y la articulación de acciones y actores para la consecución de resultados; diseñados las acciones en función a la resolución de problemas críticos que afectan a la población y que requiere de la participación de diversos actores, que alinean su accionar:

Orientado a Resultados.- vinculados a los objetivos nacionales plasmados en el Plan Estratégico Nacional. Orientado al Ciudadano.

Relación Causal de la prestación.- entre los productos entregados y los resultados 
esperados sobre la base de evidencia disponible

Visión de Proceso.- Presenta un ciclo continuo e integrado de la programación, formulación, aprobación, ejecución, contabilización y evaluación presupuestaria.

Habiéndose Sistematizado la Administración Financiera del Estado y la consiguiente Implementación del Presupuesto Por Resultados, en lo que respecta a la ejecución del Gasto se han modificado una serie de normativos de Gestión Presupuestal como es:

La Programación Presupuestaria Trimestral Mensualizada. "PPTM", creada por la cuadragésima Segunda Disposición final de la Ley Adjetiva para la programación de gastos de la fuente de financiamiento de Recursos Ordinarios y a cargo de la Dirección Nacional de Presupuesto Público. DNPP,

PCA Programación de Compromiso Anual.- Que considera la herramienta más importantes de la gestión presupuestaria en miras de alcanzar Presupuesto Por Resultados, haciendo que las decisiones administrativas de gasto sean más agiles, y de mayor alcance, pudiendo ser hasta por todo el monto del presupuesto aprobado para el año que permite como es negociar con los proveedores para suministros por todo el año. y sin la necesidad de contar con una previa autorización o visto bueno del Ministerio de Economía y Finanzas, a diferencia del anterior Calendario de Compromiso que estaba supeditados tanto las certificaciones presupuestales mensuales y por ende la gestión administrativa se encontraba muy delimitada

Calendario de Compromisos Institucional.- Calendario solamente en relación al Pago.
En tanto, se ha resumido los procesos presupuestarios de gastos con una previa:

Certificación Presupuestal.- Verificación del Crédito Presupuestal en relación de la programación de actividades por la unidad ejecutora correspondiente.

Compromiso.- Llamada también afectación, o incidencia presupuestaria (que todavía en si no es la ejecución presupuestaria) Es el acto mediante el cual se acuerda luego del cumplimiento de los tramites legalmente establecidos la realización de gastos previamente aprobados afectando total o parcialmente los créditos presupuestarios aprobados. El Compromiso Presupuestal se efectúa con posterioridad a la generación de la obligación como son los contratos de personal, órdenes de compra, ordenes de servicio, etc. Se consideran 19 comprobantes administrativos que sirven para comprometer. La incidencia se efectúa a través del SIAF

Devengado.- la adquisición misma del derecho o la obligación, sustentado con los comprobantes fiscales, también su incidencia es vía SIAF, y es carácter de Derecho Administrativo.

Pagado.- Es la Ejecución del Ingreso o derecho con la cobranza y las obligaciones que son originados en el Gasto con el Pago.

\section{PROGRAMACIÓN ESTRATEGICA PRESUPUESTARIA}

Proceso en el que se relacionan los resultados con los productos, acciones y medios necesarios para su ejecución, los cuales se basan en información y experiencia existente (evidencias). Este proceso se realiza con la finalidad de solucionar un problema crítico que afecta a una determinada población (condición de interés). 
Se aplica en las fases de programación y formulación presupuestal y comprende el diseño de estrategias e intervenciones articuladas del Estado para la generación de cambios específicos en alguna condición de bienestar del ciudadano. Estos cambios son los resultados.

El producto concreto de la aplicación de la PPE es el Programa Estratégico (PE), que bajo la óptica de mejorar el bienestar ciudadano, provee un conjunto de insumos, productos y resultados articulados causalmente. La particularidad del PE es que se diseña, en sus etapas iníciales, independientemente de los que las instituciones ya vienen haciendo, y con el objeto de definir una hoja de ruta que, bajo el enfoque del uso de evidencias, permite proveer bienes y servicios públicos para lograr el resultado deseado.

\section{PRELACION DE REGISTROS PARA LA PROGRAMACION MULTIANUAL DE INVERSION PÚBLICA}

Para establecer estrategias de acuerdo a las prioridades que tomen las altas autoridades del pliego presupuestario es necesario conocer los pasos que se sigue y las prelaciones para las programaciones de las inversiones Públicas, que comienzan en la Oficina de Programación e Inversión (OPI) de cada Institución, quien remite a la Dirección General de Programación Multianual del Sector Público (DGPM), antes de 16 de febrero de cada año, viabilizado su consistencia para registrar en el PMIP, a través del aplicativo informático en la siguiente prelación:

a. Proyectos nuevos viabilizados en el primer año del PMIP.

b. Proyectos, cuya ejecución serán financiadas con transferencias del Sector, serán registrados en PMIP de la entidad que recibe la transferencia. c. Para el registro deberán considerarse solamente aquellos que cuenten con estudio de pre inversión registrado en el Banco de Proyectos.

d. No se podrán programar proyectos de emergencia declarados elegibles en el primer semestre del año fiscal en el que se realiza la programación. E-e-Los PIP pese que cuenten con código presupuestal pero no cuenten con estudio de pre inversión y registrado en el Banco de Proyectos no se incluyen en la programación, salvo disposición legal que no requieran contar con declaración de viabilidad.

e. Los proyectos de inversión con declaración de viabilidad, incorporados en el Presupuesto Institucional de Apertura del año fiscal en el que se realiza la programación, son considerados proyectos en ejecución en el PMIP, sea que cuenten con gastos devengados o no.

Luego que la DGPM verifique la consistencia del PMIP, el Órgano Resolutivo de cada Sector debe aprobar el PMIP a más tardar el 30 de mayo de cada año y remitirlo a la DGPM.

\section{INTERACCIÓN ESTRATEGICA DE PROGRAMACIÓN DE INVERSÓN PÚBLICA}

Técnicas de planificación económica Políticas económicas de decisión Habilidades de negociación ejecutiva

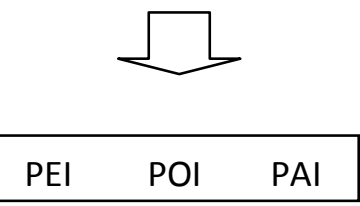


CONDICIONES PARA PROGRAMACION PLURIANUAL EFECTIVA

- Consenso de Compromiso Político

- Planificación estratégica efectiva

- Visión Prospectiva

- Normativa Técnica

- Gerencia Estratégica

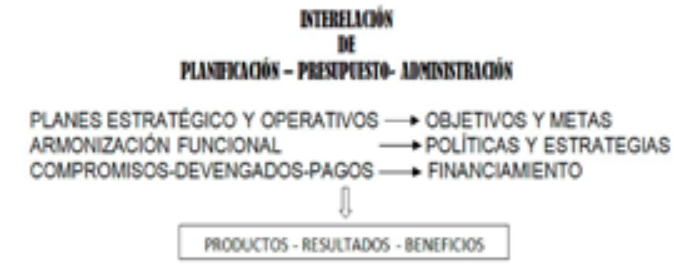

PANAROMA FABORABLE PARA

MAYOR ENFASIS DEL PpR CON PROYECCION MULTIANUAL PARA SECTOR EDUCACION

El sector Educación, ha recibido en el presente año Fiscal 13,186 millones que en relación al Producto Bruto Interno (PBI), el porcentaje destinado al sector Educación subió de 2,7 por ciento del PBI en el 2011 a 3 por ciento en el 2012. En miras de la meta del 6 por ciento del PBI para el año 2016, con el objetivo establecido por el Acuerdo Nacional. y la manifestación del Ministro de Economía y Finanzas Luis Miguel Castilla, siendo las siguientes proyecciones de los:
En tanto que habrá mayor captación de recursos ordinarios con proyección positiva de la Presión Tributaria que en relación del incremento de los Ingresos corrientes dos $0.2 \%$ del 2011 al 2012, se proyecta para el 2013 0.3\% además de medidas estratégicas que se tomen para incrementar aún más la Presión Tributaria, que también tiende a incrementarse en un $0.3 \%$ para 2013 mientras para el 2012 en relación al 2011 se mantuvo el mismo porcentaje. Así como el PBI al mes de Julio 2012 se consolidan en $7.12 \%$.

Por otra parte necesita dar mayor incentivo a la Inversión del Sector Privado en tanto tiende a reservarse para reorientar en sus ahorros como en sus ahorros; así como lo del sector Público.

\section{DINAMISAR LA APLICACIÓN DEL PRESUPUESTO POR RESULTADOS}

Mientras el panorama de los indicadores macroeconómicos tienen proyecciones favorables sin embargo las ejecuciones presupuestarias al mes de Julio 2012 el avance es muy lento aún más con las facilidades para la gestión administrativa como es la Programación de Compromisos Anuales que antes del 2011 estuvieron limitados por los Calendarios Mensuales.

\begin{tabular}{|l|c|c|r|}
\hline \multirow{2}{*}{ INDICADORES MACROECONÓMICOS } & \multicolumn{3}{|c|}{ PROYECCION PERCENTUAL } \\
\cline { 2 - 4 } & 2011 & 2012 & 2013 \\
\hline Ingresos Corrientes & 20.5 & 20.7 & 21.0 \\
Demanda Interna & 7.8 & 6.2 & 6.0 \\
Presión Tributaria & 15.3 & 15.3 & 15.6 \\
Ahorro del Sector Publico & 6.8 & 7.5 & 8.1 \\
Ahorro del Sector Privado & 17.2 & 16.3 & 16.0 \\
Inversión Sector Público & 5.6 & 5.9 & 6.1 \\
Inversión Sector Privado & 20.9 & 21.2 & 21.4 \\
\hline
\end{tabular}

Fuente BCR-MEF 
AVANCE DE LA EJECUCIÓN PRESUPUESTARIA 2012

\begin{tabular}{|c|c|c|c|l|l|c|}
\hline PIA & PIM & \multicolumn{2}{|c|}{ COMPROMISO } & DEVENGADO & PAGADO & $\begin{array}{c}\text { AVANCE } \\
\%\end{array}$ \\
\cline { 3 - 4 } & & ANUAL & MENSUAL & & & \\
\hline 96,534'635 & $117,065^{\prime} 352$, & $77,329^{\prime} 847$, & $61,635^{\prime} 823$, & $58,686^{\prime} 620$, & $57,026^{\prime} 175$, & 50.2 \\
\hline
\end{tabular}

Que pudiendo comprometerse para el año todo el Presupuesto Inicial Modificado y luego devengar de acuerdo a las gestiones financieras para efectivizar el pago aún se viene limitado al compromiso mensual casi por la misma cantidad de lo pagado.

\section{PRESUPUPUESTO POR RESULTADOS AUN EN PROGRAMAS SOCIALES SIN LOGROS}

La aplicación del PpR empezó tomando experiencia en los programas sociales principalmente en la del Salud y Nutrición. En tanto se proyecta para el año 2013:

Para sector Salud un presupuesto de S/ 8,658 'que representa el 9 por ciento del total del presupuesto y un incremento de $11.5 \%$ respecto al presente año. En ello, para el Programa de Salud Materno Neonatal S/1,400' con variación del $32 \%$ respecto a este año.

Las razones es que el promedio nacional de desnutrición crónica infantil es de 22,6 $\%$ según la Organización Mundial de Salud (OMS), lo que significa que hay más de 750 mil niños menores de cinco años con desnutrición crónica. De acuerdo al patrón NCHS, la desnutrición crónica infantil en el Perú es de 17,9 por ciento (más de 520 mil niños).

\section{CONCLUSIONES}

1. En la sistematización de la Administración Financiera del Estado según su Ley Marco, con el enfoque de carácter financista se ha considerado solamente cuatro (4) sistemas, faltando incluir otros sistemas.
2. El Sistema Nacional de Planificación aun no se encuentra operativa desde su adscripción en la Presidencia de Consejo de Ministros imposibilitando la contundente aplicación del Presupuesto por Resultado, porque no hay presupuesto sin planificación. Orientándose mayores recursos para, entre otros gastos, la inversión social, lo cual es posible en razón al crecimiento económico del país y los mayores ingresos fiscales.

\section{RECOMENDACIONES}

1. Ley Marco de la Administración Financiera del Estado necesita modificar para incluir por lo menos los Sistemas Nacional de Planificación, Sistema Nacional de Inversión Pública (SNIP) y Sistema Nacional de Control.

2. Al Sistema Nacional de Planificación debe complementarse con su implementación desde sus principios regulatorios y adscribirse al Ministerio de Economía y Finanzas como uno de los Sistemas de la Administración Financiera del Estado y con ello ponderar la aplicación del Presupuesto por Resultado. Y mientras tanto la Dirección General de Presupuesto Público debe complementar la implementación normativa del Presupuesto por Resultados en relación estratégica de la Programación de Compromisos Anuales y Programación Multianual de la Inversión Pública

3. El Ministerio de Economía y Finanzas (MEF) a través de la Dirección General 
de Presupuesto Público (DGPP) ponderé los efectos de la Reorganización declarada según Ley del Presupuesto 2010 en el marco de su atribución del perfeccionamiento y seguimiento permanente de la técnica presupuestaria por productos y resultados. Complementando estratégicamente con:

$\checkmark$ Asignar los recursos bajo el marco de las prioridades de la política pública y respetando los parámetros de la política fiscal.

$\checkmark$ Articular la asignación de recursos públicos a productos y resultados.

$\checkmark$ Ampliar la cobertura de intervenciones públicas diseñadas en base a evidencias disponibles.

$\checkmark$ Generar y usar información de desempeño para una asignación más eficaz y eficiente de los recursos públicos.

$\checkmark$ Institucionalizar la programación multianual del gasto.

$\checkmark$ Articular el gasto corriente y gasto de capital.

$\checkmark$ Lograr la articulación territorial por macro regiones.

\section{REFERENCIAS BIBLIOGRÁFICAS}

1. Constitución Política del Perú 1993. Lima-Perú

2. Marco de la Administración Financiera del Sector Público-Ley N²8112(28-112003) Lima.
3. Ley General del Sistema Nacional de Presupuesto- Ley N²8411 (25-112004) Lima- Perú

4. Ley Anual del Presupuesto Público para el año fiscal 2010 ( 17-12-2009) LimaPerú

5. Diario Gubernamental 2009- Editores Pacifico. Lima-Perú

6. Diario Gubernamental 2010- Editores Pacifico. Lima-Perú

7. Instituto Pacifico: Gobierno y Cooperación Internacional: Actualidad Gubernamental Año IV- meses Enero Febrero, Marzo, Julio, Setiembre y Diciembre 2011 Nos. 27, 28, 29, 33, 35 y 38 LimaPerú

8. Castillo Chávez J, (2004) Administración Pública - Edit. Centro de Estudios Gubernamentales Lima Perú

9. Safra Meléndez J.(2011) Agenda Gubernamental-Edit. Marketing Consultores S.A Lima Perú

10. García Riega A.- (2009) Presupuesto por Resultados y Presupuesto ParticipativoInstituto Pacifico - Lima.

11. Gestión Tributaria -Presupuesto Sector Público (2012)-Presupuesto Asignado a la Educación, UNMSM-Lima-Perú

12. http://ecoeduperu.blogspot.com/

13. http://es.scribd.com/doc/47607766/ Sistema-Nacional-de-Presupuesto 